\title{
The Increasing Relevance of Tumour Histology in Determining Oncological Outcomes in Colorectal Cancer
}

\author{
Iris D. Nagtegaal ${ }^{1} \cdot$ Niek Hugen $^{2}$ \\ Published online: 2 August 2015 \\ (C) The Author(s) 2015. This article is published with open access at Springerlink.com
}

\begin{abstract}
Colorectal cancer is not just one type of cancer. Differences in outcome and reaction to treatment can at least be partly explained by different histological and molecular subtypes. Recognition of these differences may influence treatment decisions. However, there is huge variation in the amount of information that is available. Several tumour types such as mucinous carcinoma, signet ring cell carcinoma, neuroendocrine carcinoma and adenosquamous carcinoma have such a distinct phenotype that they are readily recognised. However, due to the rarity of signet ring cell carcinoma and adenosquamous carcinoma, limited data are available. More recently defined subtypes, like medullary carcinoma, serrated adenocarcinoma and micropapillary carcinoma, are not adequately diagnosed, which limits research possibilities using large-scale data from registries. In the current review, we systematically describe the histologic subtypes with the clinical and molecular background. We evaluate their prognosis compared to adenocarcinoma not otherwise specified and speculate about the clinical relevance.
\end{abstract}

Keywords Colorectal cancer · Adenocarcinoma $\cdot$ Mucinous carcinoma $\cdot$ Signet ring cell carcinoma $\cdot$ Neuroendocrine carcinoma $\cdot$ Adenosquamous carcinoma $\cdot$ Micropapillary

This article is part of the Topical Collection on Localized Colorectal Cancer

Iris D. Nagtegaal

iris.nagtegaal@radboudumc.nl

Niek Hugen

Niek.hugen@radboudumc.nl

1 Department of Pathology, Radboudumc, PO Box 9101, 6500 HB Nijmegen, The Netherlands

2 Department of Surgery, Radboudumc, PO Box 9101, 6500 HB Nijmegen, The Netherlands carcinoma $\cdot$ Medullary carcinoma $\cdot$ Serrated carcinoma . Survival $\cdot$ Prognosis $\cdot$ Microsatellite instability $\cdot$ RAS mutation $\cdot$ BRAF mutation

\section{Introduction}

Differences in oncological outcome of cancer have been long recognised, and with the apparently limitless possibilities of next-generation sequencing, it has been shown that at least part of these differences can be ascribed to the molecular background. However, the influence of tumour microenvironment should not be ignored and might very well be responsible for the limited responsiveness to therapy in a percentage of tumours [1]. Both molecular background and tumourmicroenvironment interactions are closely associated with tumour type. This phenotypic determination of the character of tumour cells has been shown to be very relevant for prognosis. In colorectal cancer, mucinous carcinoma, the most frequent of the well-recognised subtypes, has a very limited response to systemic therapy in the metastatic setting, probably due to the distinct pattern along which the tumour disseminates [2]. Solitary liver metastases are uncommon in mucinous carcinomas, whereas peritoneal spread is more common.

Several subtypes have been recognised for a very long time, due to the clear differences in their phenotypes compared to the common adenocarcinoma (AC). Mucinous carcinoma (MC, Fig. 1a) is the most frequent phenotype in this group and therefore relatively well-studied. The other subtypes like signet ring cell carcinoma (SRCC, Fig. 1b), neuroendocrine carcinoma (NEC, Fig. 1c) and adenosquamous carcinoma (ASC, Fig. 1d) are very rare, and therefore, limited information can be gathered about their response to therapy. More recently, three additional subtypes have been defined, at least partly based on molecular and genetic studies. Medullary 
Fig. 1 Different histological subtypes of colorectal cancer. a Mucinous carcinoma (MC), b signet ring cell carcinoma (SRCC), c neuroendocrine carcinoma (NEC), $\mathbf{d}$ adenosquamous carcinoma (ASC), e medullary carcinoma $(\mathrm{MeC})$, f serrated carcinoma $(\mathrm{SeC})$ and $\mathbf{g}$ micropapillary carcinoma (MiC)

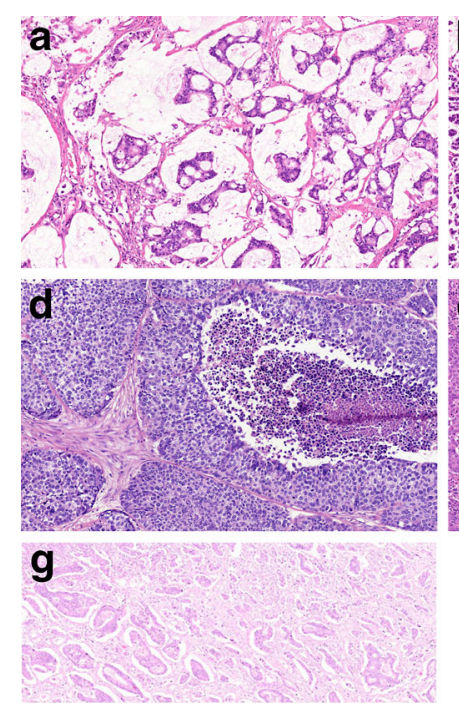

carcinoma (MeC, Fig. 1e) has a very strong correlation with microsatellite instability and Lynch syndrome, while serrated carcinoma (SeC, Fig. 1f) is strongly linked to the serrated pathway among which colorectal cancer can develop. Finally, in analogy with breast carcinoma and other tumour types, the micropapillary subtype has been recognised (MiC, Fig. 1g). In the general population, there is underreporting of these subtypes, because of insufficient recognition in daily practise. Evidence for aberrant clinical behaviour is only derived from single-centre studies and therefore needs validation in larger series.

In this review, we describe the different subtypes with the molecular background (if known) in correlation with clinical relevant issues and outcome data.

\section{Mucinous Carcinoma}

In the spectrum of colorectal cancer (CRC), $\mathrm{MC}$ is the second largest histological subtype next to AC (10-15\% of CRCs) [1]. A tumour is designated as $\mathrm{MC}$ when more than $50 \%$ of the tumour volume consists of extracellular mucus [3]. In the pools of mucus, malignant epithelium can be found in clumps of cells or as single cells [4]. The distinct histological presentation of MC has led to the hypothesis that these tumours may develop along a distinct oncogenic pathway, but exact mechanisms have not been elucidated to date. MCs have a tendency to be located in the right hemicolon (54-60\%), they present at a more advanced stage of disease, and high frequencies of MC are observed in Lynch syndrome patients (22-40 \%) [1, 5-7]. The latter also explains the higher rate of microsatellite instability (MSI) that is seen in MC [7]. However, when MCs do develop according to the chromosomal instability pathway, they present with a markedly lower rate of chromosomal instability compared with ACs [8]. Other common molecular aberrations in $\mathrm{MC}$ are the higher rates of $K R A S, B R A F$ and $P I 3 K$ mutations, when compared with AC [7]. Constitutive activation of the RAS/RAF/MAPK and PI3K/AKT signalling pathways influences cell growth, survival, proliferation and cell motility, thus influencing tumour behaviour.

Since MC is diagnosed approximately once in every eight CRC patients, the prognostic value of this subtype has been studied extensively, but discussion remains. MC in general has long been considered an unfavourable prognostic indicator, but this has been disputed in various studies recently. In this perspective, the importance of the location of the primary tumour has been highlighted, since treatment strategies and prognosis vary for colon and rectal cancer patients.

For colonic MC, large population-based studies have demonstrated that there is no difference in overall survival after correction for stage at presentation [1, 5, 9]. Moreover, in a recent retrospective cohort study of 435 non-metastatic patients who were diagnosed with CRC between 2000 and 2010, it was demonstrated that $\mathrm{MC}$ was associated with an improved outcome (HR 0.75; $95 \%$ CI 0.46-1.21). This favourable outcome has not been reported for rectal MC to date, and the controversy regarding prognosis in rectal MC patients has been ongoing over the past few decades. Rectal MCs tend to respond poorly to neoadjuvant therapies such as chemoradiotherapy, and high rates of incomplete resections have been reported $[10,11,12 \cdot \bullet]$. However, when adequate circumferential resection margins can be obtained during surgery, there is no difference in local recurrence rate and overall survival between rectal MC and AC [13]. Multidisciplinary assessment of rectal cancer patients including accurate imaging, optimal preoperative therapy and high-quality total mesorectal excision surgery has demonstrated to improve outcome for rectal MC [13] (Table 1).

As mentioned afore, MCs present at a higher stage of disease compared with ACs. Between 19 and $29 \%$ of MC 
Table 1 Overview of studies that analysed overall survival of colorectal SRCC patients compared with AC patients using multivariable or univariable analysis

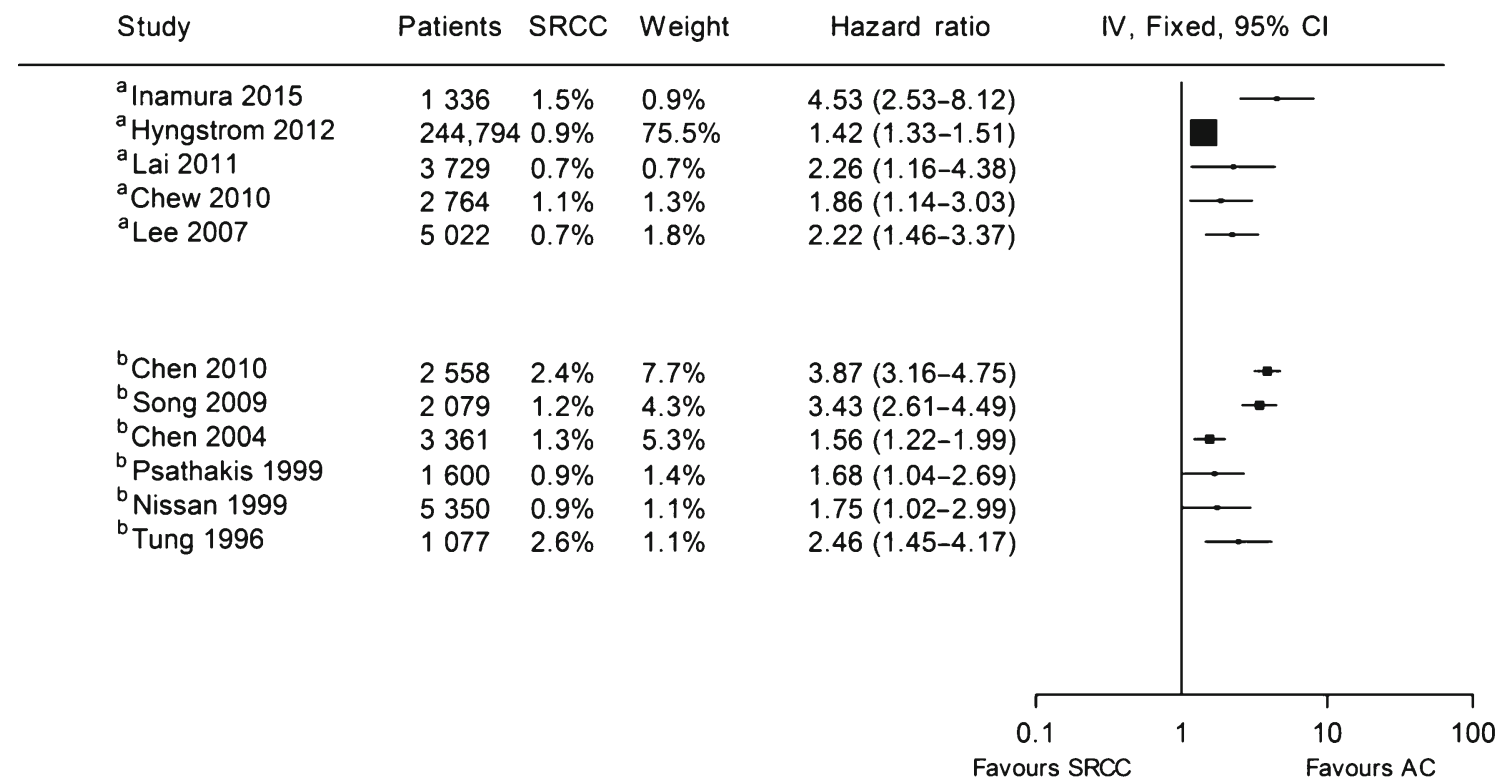

Hazard ratios as reported in studies were used for analysis. If no hazard ratio was reported, it was calculated from the published data as described by Parmar et al. [69]

${ }^{\mathrm{a}}$ Multivariable analysis

${ }^{\mathrm{b}}$ Univariable analysis

patients have metastatic disease upon their first presentation, and MCs are more likely to develop metastases during followup $[1,2,5]$. Although mechanisms are unclear, it has been demonstrated that $\mathrm{MC}$ has a distinct metastatic pattern of spread than AC. MC patients more frequently have metastatic disease in more than one organ. Compared with $\mathrm{AC}$, hepatic metastases are less common in MC, and especially peritoneal metastases are frequently seen [2].

The deviant metastatic pattern of MCs is considered one of the possible explanations for the poor outcome after palliative chemotherapy as observed in various studies [12••, 14-17]. Median overall survival rates for MC patients from these studies are approximately 12 months, suggesting a reduced efficacy of chemotherapy for advanced-stage MC [12••]. In the adjuvant setting, no difference in outcome following chemotherapy has been observed when compared with AC $[9,12 \bullet, 18]$.

\section{Signet Ring Cell Carcinoma}

Signet ring cell carcinoma (SRCC) is a rare subtype (1\% of $\mathrm{CRCs}$ ) and is defined by the presence of the typical signet ring cells that comprise at least half of the tumour volume $[1,3]$. Signet ring cells get their typical appearance as a result of a large mucus vacuole that displaces the nucleus to the edge of the cell. Compared with AC patients, SRCC patients are generally younger. Over $25 \%$ of SRCC patients is under the age of 60 [1]. As for MC, SRCC is commonly found in the proximal (right) hemicolon in more than half of the patients $[1,19]$. This corroborates very well with the high rate of MSI that is seen in SRCC tumours (24-40 \%) [20, 21]. Especially SRCCs that also present with extensive mucus surrounding the tumour are likely MSI [20]. SRCCs are frequently of the CpG island methylator phenotype ( $48 \%$ ), and $B R A F$ mutations are found in $30-33 \%$ of SRCCs [20, 21]. KRAS is mutated in $53 \%$ of SRCCs [21]. SRCCs are notorious for their rapid progression to an advanced stage of disease and are more likely to present with locally advanced and lymph nodepositive tumours, with over three quarter of tumours being stage III or IV at the time of diagnosis $[1,5]$. Perineural growth and lymphatic and vascular invasion are frequently present [20].

Approximately one third of SRCC patients already has metastatic disease upon the first presentation, and SRCC patients are more likely to develop metastases during follow-up $[1,2,5]$. SRCC has a distinct metastatic pattern, in which metastases develop at multiple sites $(71 \%)$. Liver metastases only account for $32 \%$ of metastases in SRCC patients, and metastases to the peritoneum are seen in $51 \%$, compared with 73 and $20 \%$, respectively, in AC patients [2]. Especially peritoneal metastases in CRC are clinically challenging, but the introduction of cytoreductive surgery followed by hyperthermic intraperitoneal chemotherapy (HIPEC) has shown promising results and has improved prognosis for this patient group [22]. Unfortunately, for SRCC patients, a high rate of 
recurrence after HIPEC has been shown, resulting in a meagre median survival reaching only slightly over 1 year [23•, 24]. This had led to the recommendation to refrain from aggressive therapeutic approaches for peritoneal SRCC metastases in the presence of other poor prognostic factors [23•].

Prognosis for SRCC patients is not only poor for advancedstage disease. SRCCs have stage-independent poorer outcomes when compared with AC [5, 25-33]. A study from 2015 showed that poorer outcomes were seen in both colon and rectal cancer patients separately, with 5-year relative survival rates of 30.8 and $19.5 \%$, respectively [1].

Despite the poor reported outcome, the clinical subset of SRCC patients is too small to being addressed in clinical studies, rendering insight into response to therapies limited. Although a poor outcome for metastatic patients following cytoreductive surgery and HIPEC has been described, benefit of adjuvant chemotherapy seems comparable with AC [1]. Additional analyses are highly needed and may give more direction in therapeutic strategies.

\section{Neuroendocrine Carcinoma}

Neuroendocrine carcinomas form the end of a spectrum which ranges from adenocarcinomas with neuroendocrine differentiation via MANEC (mixed adenoneuroendocrine carcinoma) to NEC. With the introduction of immunochemistry and antibodies that were specific for neuroendocrine differentiation, large series of adenocarcinomas have been analysed. For the current review, we exclude the carcinoids, but in many studies, these are analysed in combination with NEC.

Differentiated neuroendocrine cells have been detected either scattered through the tumour or in distinct nests in approximately $20 \%$ of adenocarcinomas [34]. When applying a definition of less than $30 \%$ of neuroendocrine differentiation in tumour, a recent metaanalysis [34] including 1587 patients from 11 studies shows that neuroendocrine differentiation in adenocarcinomas is associated with a decreased 5-year survival rate (pooled OR 0.60, $95 \%$ CI $0.37-0.97$ ). This is in line with the recent publication of Shafqat et al. [35•].

MANEC was first defined in the 2010 edition of the WHO Blue Book [3] and describes tumours with both adenocarcinoma and neuroendocrine carcinoma components that each has a proportion of at least $30 \%$. Current literature is mainly limited to case reports. One study [36] compared 12 patients with MANEC with 27 patients with NEC and did not find any differences in survival.

There has been an increase in the incidence of colorectal neuroendocrine tumours over recent years [35•]; however, these tumours still account for a very small contingent of colorectal carcinomas, less than $1 \%$. From studies that compared the small adenocarcinoma components of these tumours with the neuroendocrine components [37-39], we know that both components have a clonal relationship, which can be traced back to the adenomatous stage. Mutational status (KRAS, BRAF, NRAS, PIK3CA, p53) in these tumours is not different from adenocarcinomas [39].

Compared to high-grade AC [35॰], patients with NEC are younger, more often male. Tumours present as metastatic disease, and the primary location is more often in the rectum. In this large registry-based analysis, the 5 -year overall survival of patients with NEC $(n=1367)$ was very poor, even compared with high-grade AC $(n=$ $72,553), 16.3$ versus $50.2 \%$. Similar data are obtained in an earlier SEER-derived study, where 455 NECs identified between 1992 and 2000 with a relative 5year survival of $21.4 \%$ were compared to all adenocarcinomas (5-year relative survival $62.1 \%$ ) [40]. This is in line with the data obtained from the NORDIC NEC study [41], where NECs derived from the colon and rectum demonstrate the worst survival rates of all NECs of the gastrointestinal tract.

\section{Adenosquamous Carcinoma}

Like NEC, ASC is also part of a spectrum, which ranges from adenoacanthoma (i.e. adenocarcinomas with benignappearing squamous metaplasia) through ASC to pure squamous carcinoma. The first and the latter are extremely rare. Cancer registries estimate the incidence of ASC between 0.06 and $0.09 \%[42,43]$. Patient characteristics are not different from adenocarcinomas, but these tumours tend to present in advanced stages [42] and are located more often in the proximal and transverse colon, compared to AC. When they are located in the rectosigmoid area, the prognosis seems slightly better [43]. In early-stage disease (pT1-3N0), outcomes are comparable to $\mathrm{AC}$; however, all other tumours have a significantly worse outcome, which is most pronounced in patients with synchronous metastases. Five-year overall survival is $23.5-25.4 \%$ compared to $41.6-53.6 \%$ in AC $[42,43]$. However, these data are based on a total number of 244 patients. Molecular data and information about treatment response are not available.

\section{Medullary Carcinoma}

$\mathrm{MeC}$, previously known as solid-type poorly differentiated carcinoma and large cell minimally differentiated carcinoma, is characterised by sheets of malignant cells with vesicular nuclei, prominent nucleoli and abundant eosinophilic cytoplasm, exhibiting prominent infiltration by intraepithelial lymphocytes [3]. Single-centre studies estimate its frequency at approximately $4 \%$ [44-46]. It is assumed that this type is 
underreported in cancer registries $(0.08 \%)$ [47], since it might be difficult to distinguish from poorly differentiated adenocarcinoma [48]. This subtype can be difficult to recognise, especially on preoperative biopsies, because of the undifferentiated appearance and the aberrant immunohistochemistry. Because of the lack of staining with cdx2 [49] and cytokeratin 20, it has been suggested that these tumours lose their intestinal differentiation. However, other intestinal markers, such as MUC1, MUC3 and TFF3, can still be demonstrated in $\mathrm{MeC}$ [50].

$\mathrm{MeC}$ is more frequent in female patients $[45,51]$ and has a very strong correlation with age, with an increased frequency in the elderly [51]. The preferred location is the right colon [44, 45]. These tumours are larger in size [44, 45], and upon diagnosis, it is hard to detect premalignant mucosa [44]. Compared to poorly differentiated adenocarcinoma, less nodal positivity and less extramural vascular invasion (EMVI) are present [44]. Compared to AC, more frequent lymphovascular invasion (LVI) is detected (62.9 versus $36.5 \%$ ) [45]. This subtype is invariably associated with MSI and a very high rate of BRAF mutations: 86 versus $69 \%$ in other MSI tumours and $19 \%$ or less in the general population [45].

In general, the prognosis is very good, compared to adenocarcinoma [44, 52]. The study of Knox et al. [45] failed to show this favourable prognosis in the univariate analysis, due to an unexplained high 30-day mortality in this group, but in the multivariate analysis there is an improved survival (HR $0.54,95 \%$ CI $0.30-0.96)$. There are no data about treatment response.

\section{Serrated Adenocarcinoma}

The recognition of the serrated pathway as an important developmental route towards CRC has led to the proposition of SeC. This subtype is characterised by architectural similarity to sessile serrated lesions, with glandular serration, that may be accompanied by mucinous areas. The cells have a low nucleus-to-cytoplasm ratio [3]. However, all the available literature is from the same group of investigators, which limits the generalisability of the data. The data concerning $\mathrm{SeC}$ are lacking behind the enormous amount of information we are gathering about the serrated polyp [53].

Approximately $10 \%$ of all CRCs can be classified as $\mathrm{SeC}$ [54]. One of the remarkable but also confusing features of $\mathrm{SeC}$ is that both a mucinous and a non-mucinous subtype exist. Actually, up to $45 \%$ of all $\mathrm{SeC}$ occur in mucinous carcinomas [55]. In general, these tumours are more often proximally located [54] and have similar stage distribution when compared to AC. Microsatellite instability rates are not different from $\mathrm{AC}$ [55], but BRAF mutations are very frequent (33.3 versus $0 \%$ ) [56]. In addition, KRAS mutations are frequently observed (45.2 versus $27.1 \%$ ) [56]. These mutation rates are similar to the ones observed in serrated polyps. In the $\mathrm{SeC}$, there is co-occurrence of KRAS mutations and MSI, which is absent in AC. When performing unsupervised clustering of expression data of $\mathrm{SeC}$ and $\mathrm{AC}$, over 200 genes are differentially expressed [57], and almost perfect separation occurs. Large-scale microarray studies suggest that a molecularly defined subtype called CSS3 exists that might be exemplary for this subtype [58]. However, the proper histological classification of the tumours in this study has not been performed. A lack of response to anti-EGFR therapy independent of KRAS status has been suggested based on in vitro experiments [58]. The (unclassified) adenocarcinomas in this molecular subgroup also have a decreased prognosis; however, in the three available series of $\mathrm{SeC}$, no difference in survival compared to $\mathrm{AC}$ has been observed [54, 57, 59].

\section{Micropapillary Carcinoma}

This tumour is characterised by small clusters of tumour cells within stromal spaces mimicking vascular channels [3]. Initially described in breast cancer, the subtype has been recognised in other tumour types as well. The micropapillary component can be recognised in both $\mathrm{AC}$ as $\mathrm{MC}$, and most studies define their tumour as MiC when they have at least a $5 \%$ component.

The incidence of this component varies between 5 and $20 \%$ of all colorectal carcinomas [60-62]. Data from cancer registries are not available. The age and gender of the patients are not different from AC, and location within the colon is also comparable [60-62]. Not all studies agree about the increased $\mathrm{T}$ stage which is sometimes described [60-62], but in all populations, the percentage of node-positive patients is high, up to $80 \%$, compared to an average of $40 \%$ in the $\mathrm{AC}$ patients [60-64]. This is in accordance with almost double the incidence of LVI [60-62, 64]. EMVI is increased (46.7 versus $19.1 \%$ ), as is perineural invasion (PNI) $[60,63]$. The risk of both positive nodes and LVI increases with an increasing percentage of the micropapillary component [60-62].

A number of studies have examined the molecular background of this new subtype. Microsatellite instability is not different from $\mathrm{AC}[61,64]$ or possibly somewhat less frequent [60]. There is no difference in mutational spectrum (p53, KRAS, BRAF) [60]. The prognosis of this group of patients is worse $[61,63,65]$. The study of Lee [61] evaluated the effects of chemotherapy in $34 \mathrm{MiC}$ patients, of which 27 were treated with systemic therapy, and in the survival curves, no 
Table 2 Summary of the different subtypes in comparison with adenocarcinoma n.o.s.

\begin{tabular}{lllllllllllll}
\hline Type & Incidence (\%) & Age & Gender & T & $\mathrm{N}$ & $\mathrm{M}$ & LVI & EMVI & MSI & RAS/RAF & Therapy & Survival \\
\hline MC & 10 & $=$ & Female & $\uparrow$ & $\uparrow$ & $\uparrow$ & $?$ & $?$ & $\uparrow$ & $\uparrow$ & $=/ \downarrow$ \\
SRCC & 1 & $\downarrow$ & $=$ & $\uparrow$ & $\uparrow$ & $\uparrow$ & $\uparrow$ & $\uparrow$ & $\uparrow$ & $\uparrow$ & $=$ \\
NEC ${ }^{\mathrm{a}}$ & $<1$ & $\downarrow$ & Male & $?$ & $\downarrow$ & $\uparrow$ & $?$ & $?$ & $=$ & $=$ & Poor \\
$\mathrm{ASC}$ & $<0.1$ & $=$ & $=$ & $\uparrow$ & $\uparrow$ & $\uparrow$ & $?$ & $?$ & $?$ & $?$ & Poor \\
$\mathrm{MeC}$ & 4 & $\uparrow$ & Female & $\uparrow$ & $\downarrow$ & $\downarrow$ & $\uparrow$ & $\downarrow$ & $\uparrow \uparrow$ & BRAF & $?$ \\
$\mathrm{SeC}$ & 10 & $=$ & Female & $=$ & $=$ & $=$ & $?$ & $?$ & $=$ & $\uparrow$ & Poor & GEGFR? \\
$\mathrm{MiC}$ & 20 & $=$ & $=$ & $\uparrow$ & $\uparrow$ & $\uparrow$ & $\uparrow$ & $\uparrow$ & $=$ & $=$ & & $?$ \\
\hline
\end{tabular}

$M C$ mucinous carcinoma, SRCC signet ring cell carcinoma, $N E C$ neuroendocrine carcinoma, $A S C$ adenosquamous carcinoma, $M i C$ micropapillary carcinoma, $\mathrm{MeC}$ medullary carcinoma, $\mathrm{SeC}$ serrated carcinoma, $L V I$ lymphovascular invasion, $E M V I$ extramural vascular invasion, aEGFR anti-EGFR therapy, $\uparrow$ higher, $\downarrow$ lower, = equal/similar, ? unknown

${ }^{a}$ NECs are compared with high-grade adenocarcinomas

difference can be observed. However, due to small numbers, no conclusions can be drawn. The decreased prognosis in combination with the sometimes observed high-grade cytologic features [60] has led to the suggestion that this tumour subtype should be graded as poorly differentiated [66] (Table 2).

\section{Conclusions}

There is more than one type of CRCs. Increasing recognition of different developmental pathways important in both carcinogenesis and treatment response has led to a renewed interest in histological subtypes. While the molecular background of tumours is extremely important, the interaction with the microenvironment should not be forgotten. The presence of an extensive inflammatory infiltrate, as can be observed in $\mathrm{MeC}$, is strongly associated with a very good prognosis [67•]. Indeed, we do observe a good outcome in this patient group, despite advanced $\mathrm{T}$ stage and increased presence of LVI. That there is more than the molecular background of tumours is also observed in MiC. Mutational status of these tumours seems comparable to $\mathrm{AC}$; however, prognosis is significantly decreased, possibly due to the high frequency of LVI and EMVI, in combination with very high percentages of lymph node metastases.

Unfortunately, with the exception of MC, we have little data on response rates to chemotherapy in these subpopulations and hence very limited data about optimal treatment regimens. Careful histological and molecular analysis of tumours in clinical trials is required to fill this gap [68]. In addition, pathologists have to provide this information in the multidisciplinary meetings and explain the possible impact of these different subtypes properly. The issue of the different histologies of colorectal cancer should be put on the agenda of future oncology meetings to raise awareness on the different response rates, natural history and outcomes of the individual types.

\section{Compliance with Ethics Guidelines}

Conflict of Interest Iris D. Nagtegaal and Niek Hugen declare that they have no conflict of interest.

Human and Animal Rights and Informed Consent This article does not contain any studies with human or animal subjects performed by any of the authors.

Open Access This article is distributed under the terms of the Creative Commons Attribution 4.0 International License (http:// creativecommons.org/licenses/by/4.0/), which permits unrestricted use, distribution, and reproduction in any medium, provided you give appropriate credit to the original author(s) and the source, provide a link to the Creative Commons license, and indicate if changes were made.

\section{References}

Papers of particular interest, published recently, have been highlighted as:

- Of importance

•- Of major importance

1. Hugen $\mathrm{N}$ et al. Colorectal signet-ring cell carcinoma: benefit from adjuvant chemotherapy but a poor prognostic factor. Int $\mathrm{J}$ Cancer. 2015;136(2):333-9.

2. Hugen $\mathrm{N}$ et al. Metastatic pattern in colorectal cancer is strongly influenced by histological subtype. Ann Oncol. 2014;25(3):651-7.

3. Bosman FT, World Health Organization, International Agency for Research on Cancer. World Health Organization classification of tumours, WHO classification of tumours of the digestive system. 4th ed. Lyon: International Agency for Research on Cancer; 2010. 417 p.

4. Nagtegaal I et al. Morphological changes in tumour type after radiotherapy are accompanied by changes in gene expression profile but not in clinical behaviour. J Pathol. 2004;204(2):183-92. 
5. Hyngstrom JR et al. Clinicopathology and outcomes for mucinous and signet ring colorectal adenocarcinoma: analysis from the National Cancer Data Base. Ann Surg Oncol. 2012;19(9):2814-21.

6. Hugen $\mathrm{N}$ et al. Insight into mucinous colorectal carcinoma: clues from etiology. Ann Surg Oncol. 2014;21(9):2963-70.

7. Hugen $\mathrm{N}$ et al. The molecular background of mucinous carcinoma beyond MUC2. J Path: Clin Res. 2014;1:3-17.

8. Hugen $\mathrm{N}$ et al. Reduced rate of copy number aberrations in mucinous colorectal carcinoma. Oncotarget. 2015.

9. Hugen $\mathrm{N}$ et al. Prognosis and value of adjuvant chemotherapy in stage III mucinous colorectal carcinoma. Ann Oncol. 2013;24(11): 2819-24.

10. Oberholzer $\mathrm{K}$ et al. Rectal cancer: mucinous carcinoma on magnetic resonance imaging indicates poor response to neoadjuvant chemoradiation. Int J Radiat Oncol Biol Phys. 2012;82(2):842-8.

11. Yu SK et al. Magnetic resonance imaging defined mucinous rectal carcinoma is an independent imaging biomarker for poor prognosis and poor response to preoperative chemoradiotherapy. Eur $\mathrm{J}$ Cancer. 2014.

12.• Hugen $\mathrm{N}$ et al. Advances in mucinous colorectal cancer patient care. Nat Rev Clin Oncol. 2015. Timely review of the improvements that have been made in the treatment of this subtype in the multidisciplinary setting.

13. Hugen $\mathrm{N}$ et al. Modern treatment of rectal cancer closes the gap between common adenocarcinoma and mucinous carcinoma. Ann Surg Oncol. 2015

14. Negri FV et al. Mucinous histology predicts for reduced fluorouracil responsiveness and survival in advanced colorectal cancer. Ann Oncol. 2005;16(8):1305-10.

15. Catalano Vet al. Mucinous histology predicts for poor response rate and overall survival of patients with colorectal cancer and treated with first-line oxaliplatin- and/or irinotecan-based chemotherapy. Br J Cancer. 2009;100(6):881-7.

16. Maisano R et al. Mucinous histology of colon cancer predicts poor outcomes with FOLFOX regimen in metastatic colon cancer. $\mathrm{J}$ Chemother. 2012;24(4):212-6.

17. Mekenkamp LJ et al. Mucinous adenocarcinomas: poor prognosis in metastatic colorectal cancer. Eur J Cancer. 2012;48(4):501-9.

18. Catalano V et al. Prognosis of mucinous histology for patients with radically resected stage II and III colon cancer. Ann Oncol. 2012;23(1):135-41.

19. Nitsche U et al. Mucinous and signet-ring cell colorectal cancers differ from classical adenocarcinomas in tumor biology and prognosis. Ann Surg. 2013;258(5):775-83.

20. Hartman DJ et al. Signet ring cell colorectal carcinoma: a distinct subset of mucin-poor microsatellite-stable signet ring cell carcinoma associated with dismal prognosis. Am J Surg Pathol. 2013;37(7):969-77.

21. Kakar S et al. Loss of heterozygosity, aberrant methylation, BRAF mutation and KRAS mutation in colorectal signet ring cell carcinoma. Mod Pathol. 2012;25(7):1040-7.

22. Verwaal VJ et al. 8-year follow-up of randomized trial: cytoreduction and hyperthermic intraperitoneal chemotherapy versus systemic chemotherapy in patients with peritoneal carcinomatosis of colorectal cancer. Ann Surg Oncol. 2008;15(9):2426-32.

23. van Oudheusden TR et al. Poor outcome after cytoreductive surgery and HIPEC for colorectal peritoneal carcinomatosis with signet ring cell histology. J Surg Oncol. 2015;111(2):237-42. Description of the effects of new treatment strategies in rare cancer types.

24. Winer J et al. Impact of aggressive histology and location of primary tumor on the efficacy of surgical therapy for peritoneal carcinomatosis of colorectal origin. Ann Surg Oncol. 2014;21(5):1456-62.

25. Inamura $\mathrm{K}$ et al. Prognostic significance and molecular features of signet-ring cell and mucinous components in colorectal carcinoma. Ann Surg Oncol. 2015;22(4):1226-35.
26. Lai $\mathrm{CC}$ et al. Low preoperative serum albumin in colon cancer: a risk factor for poor outcome. Int $\mathrm{J}$ Colorectal Dis. 2011;26(4):473-81.

27. Chew $\mathrm{MH}$ et al. Critical analysis of mucin and signet ring cell as prognostic factors in an Asian population of 2,764 sporadic colorectal cancers. Int J Colorectal Dis. 2010;25(10):1221-9.

28. Lee WS et al. Treatment outcomes in patients with signet ring cell carcinoma of the colorectum. Am J Surg. 2007;194(3):294-8.

29. Chen JS et al. Clinical outcome of signet ring cell carcinoma and mucinous adenocarcinoma of the colon. Chang Gung Med J. 2010;33(1):51-7.

30. Song $\mathrm{W}$ et al. Clinicopathologic features and survival of patients with colorectal mucinous, signet-ring cell or non-mucinous adenocarcinoma: experience at an institution in southern China. Chin Med J. 2009;122(13):1486-91.

31. Psathakis D et al. Ordinary colorectal adenocarcinoma vs. primary colorectal signet-ring cell carcinoma: study matched for age, gender, grade, and stage. Dis Colon Rectum. 1999;42(12):1618-25.

32. Nissan A et al. Signet-ring cell carcinoma of the colon and rectum: a matched control study. Dis Colon Rectum. 1999;42(9):1176-80.

33. Tung SY, Wu CS, Chen PC. Primary signet ring cell carcinoma of colorectum: an age- and sex-matched controlled study. Am J Gastroenterol. 1996;91(10):2195-9.

34. Zeng YJ et al. Prognostic significance of neuroendocrine differentiation in colorectal adenocarcinoma after radical operation: a metaanalysis. J Gastrointest Surg. 2014;18(5):968-76.

35. Shafqat $\mathrm{H}$ et al. Survival of patients with neuroendocrine carcinoma of the colon and rectum: a population-based analysis. Dis Colon Rectum. 2015;58(3):294-303. Large scale analysis of neuroendocrine colorectal carcinomas.

36. La Rosa $\mathrm{S}$ et al. Colorectal poorly differentiated neuroendocrine carcinomas and mixed adenoneuroendocrine carcinomas: insights into the diagnostic immunophenotype, assessment of methylation profile, and search for prognostic markers. Am J Surg Pathol. 2012;36(4):601-11

37. Vortmeyer $\mathrm{AO}$ et al. Concordance of genetic alterations in poorly differentiated colorectal neuroendocrine carcinomas and associated adenocarcinomas. J Natl Cancer Inst. 1997;89(19):1448-53.

38. Karkouche $\mathrm{R}$ et al. Colorectal neuroendocrine carcinomas and adenocarcinomas share oncogenic pathways. A clinico-pathologic study of 12 cases. Eur J Gastroenterol Hepatol. 2012;24(12): 1430-7

39. Kleist $\mathrm{B}$ et al. Comparison of neuroendocrine differentiation and KRAS/NRAS/BRAF/PIK3CA/TP53 mutation status in primary and metastatic colorectal cancer. Int J Clin Exp Pathol. 2014;7(9): 5927-39.

40. Kang $\mathrm{H}$ et al. Rare tumors of the colon and rectum: a national review. Int J Colorectal Dis. 2007;22(2):183-9.

41. Sorbye $\mathrm{H}$ et al. Predictive and prognostic factors for treatment and survival in 305 patients with advanced gastrointestinal neuroendocrine carcinoma (WHO G3): the NORDIC NEC study. Ann Oncol. 2013;24(1):152-60.

42. Masoomi $\mathrm{H}$ et al. Population-based evaluation of adenosquamous carcinoma of the colon and rectum. Dis Colon Rectum. 2012;55(5): 509-14

43. Cagir B et al. Adenosquamous carcinoma of the colon, rectum, and anus - epidemiology, distribution, and survival characteristics. Dis Colon Rectum. 1999;42(2):258-63.

44. Lanza $\mathrm{G}$ et al. Medullary-type poorly differentiated adenocarcinoma of the large bowel: a distinct clinicopathologic entity characterized by microsatellite instability and improved survival. J Clin Oncol. 1999;17(8):2429-38.

45. Knox RD et al. Medullary colorectal carcinoma revisited: a clinical and pathological study of 102 cases. Ann Surg Oncol. 2015.

46. Alexander $\mathrm{J}$ et al. Histopathological identification of colon cancer with microsatellite instability. Am J Pathol. 2001;158(2):527-35. 
47. Thirunavukarasu $P$ et al. Medullary carcinoma of the large intestine: a population based analysis. Int J Oncol. 2010;37(4):901-7.

48. Fiehn AM et al. Medullary carcinoma of the colon: can the undifferentiated be differentiated? Virchows Arch. 2015;466(1):13-20.

49. Hinoi $\mathrm{T}$ et al. Loss of CDX2 expression and microsatellite instability are prominent features of large cell minimally differentiated carcinomas of the colon. Am J Pathol. 2001;159(6):2239-48.

50. Winn B et al. Differentiating the undifferentiated: immunohistochemical profile of medullary carcinoma of the colon with an emphasis on intestinal differentiation. Hum Pathol. 2009;40(3):398-404.

51. Arai T et al. Hypermethylation of the hMLH1 promoter with absent hMLH1 expression in medullary-type poorly differentiated colorectal adenocarcinoma in the elderly. Mod Pathol. 2004;17(2):172-9.

52. Wick MR et al. Sporadic medullary carcinoma of the colon: a clinicopathologic comparison with nonhereditary poorly differentiated enteric-type adenocarcinoma and neuroendocrine colorectal carcinoma. Am J Clin Pathol. 2005;123(1):56-65.

53. Kahi CJ. How does the serrated polyp pathway alter CRC screening and surveillance? Dig Dis Sci. 2015;60(3):773-80.

54. Garcia-Solano J et al. Clinicopathologic study of 85 colorectal serrated adenocarcinomas: further insights into the full recognition of a new subset of colorectal carcinoma. Hum Pathol. 2010;41(10): 1359-68.

55. Tuppurainen $\mathrm{K}$ et al. Morphology and microsatellite instability in sporadic serrated and non-serrated colorectal cancer. J Pathol. 2005;207(3):285-94.

56. Stefanius $\mathrm{K}$ et al. Frequent mutations of KRAS in addition to BRAF in colorectal serrated adenocarcinoma. Histopathology. 2011;58(5): 679-92.

57. Laiho $\mathrm{P}$ et al. Serrated carcinomas form a subclass of colorectal cancer with distinct molecular basis. Oncogene. 2007;26(2): 312-20.

58. De Sousa EMF et al. Poor-prognosis colon cancer is defined by a molecularly distinct subtype and develops from serrated precursor lesions. Nat Med. 2013;19(5):614-8.
59. Makinen MJ et al. Colorectal carcinoma associated with serrated adenoma-prevalence, histological features, and prognosis. J Pathol. 2001;193(3):286-94.

60. Verdu $\mathrm{M}$ et al. Clinicopathological and molecular characterization of colorectal micropapillary carcinoma. Mod Pathol. 2011;24(5): 729-38.

61. Lee HJ et al. Colorectal micropapillary carcinomas are associated with poor prognosis and enriched in markers of stem cells. Mod Pathol. 2013;26(8):1123-31.

62. Haupt $\mathrm{B}$ et al. Colorectal adenocarcinoma with micropapillary pattern and its association with lymph node metastasis. Mod Pathol. 2007;20(7):729-33.

63. $\mathrm{Xu} \mathrm{F}$ et al. Micropapillary component in colorectal carcinoma is associated with lymph node metastasis in T1 and T2 stages and decreased survival time in TNM stages I and II. Am J Surg Pathol. 2009;33(9):1287-92.

64. Kim MJ et al. Invasive colorectal micropapillary carcinoma: an aggressive variant of adenocarcinoma. Hum Pathol. 2006;37(7): 809-15.

65. Lino-Silva LS, Salcedo-Hernandez RA, Caro-Sanchez CH. Colonic micropapillary carcinoma, a recently recognized subtype associated with histological adverse factors: clinicopathological analysis of 15 cases. Color Dis. 2012;14(9):e567-72.

66. Barresi $\mathrm{V}$ et al. Micropapillary pattern and poorly differentiated clusters represent the same biological phenomenon in colorectal cancer: a proposal for a change in terminology. Am J Clin Pathol. 2014;142(3):375-83.

67. Galon J et al. Towards the introduction of the 'Immunoscore' in the classification of malignant tumours. J Pathol. 2014;232(2):199 209. Demonstrates the importance of tumor microenvironment and explores the possibilities of implementation of this new diagnostic approach.

68. Nagtegaal ID et al. Pathology is a necessary and informative tool in oncology clinical trials. J Pathol. 2014;232(2):185-9.

69. Parmar MK, Torri V, Stewart L. Extracting summary statistics to perform meta-analyses of the published literature for survival endpoints. Stat Med. 1998;17(24):2815-34. 